\title{
Injection of HGF Plasmid cDNA to Prevent Manifestation of Parkinson Disease: A Preclinical Study using a Primate Model
}

\author{
Hiromi Koike $^{1,2}$, Akihiko Ishida ${ }^{1,2, *}$, Takuya Hayashi ${ }^{3}$, Munehisa Shimamura ${ }^{1,4}$, Shinya Mizuno ${ }^{5}$ \\ Toshikazu Nakamura ${ }^{5}$, Hidehiro Iida ${ }^{3}$, Toshio Ogihara ${ }^{6}$, Yasufumi Kaneda ${ }^{7}$ and Ryuichi Morishita ${ }^{1}$
}

\begin{abstract}
${ }^{1}$ Division of Clinical Gene Therapy, ${ }^{2}$ AnGes MG, Inc., Ibaraki, Osaka, Japan, ${ }^{3}$ Department of Investigative Radiology, National Cardiovascular Center, Research Institute, Suita, Osaka, Japan, ${ }^{4}$ Department of Advanced Clinical Science and Therapeutics, Tokyo University Graduate School of Medicine, Hongo, Tokyo, Japan, ${ }^{5}$ Department of Biochemistry and Molecular Biology, ${ }^{6}$ Department of Geriatric Medicine, ${ }^{7}$ Division of Gene Therapy Science, Graduate School of Medicine, Osaka University, 2-2 Yamada-oka, Suita, Osaka, Japan
\end{abstract}

\begin{abstract}
Parkinson disease (PD) is a neurodegenerative disorder characterized by the loss of dopaminergic neurons. Current therapies provide symptomatic treatment only and thus fail to prevent the process of neurodegeneration. Recently, it has been reported that neurotrophic factors could support the survival and enhance the function of dopaminergic neurons. Thus, gene therapy using neurotrophic factors has become the center of interest. From this viewpoint, we focused on hepatocyte growth factor (HGF) as a novel neurotrophic and angiogenic growth factor. In this study, we examined the effects of over-expression of HGF on clinical symptoms induced in a monkey model of PD, aiming toward human gene therapy. Stereotaxic transfection of naked plasmid DNA encoding human HGF cDNA into the striatum resulted in significant prevention of behavioral, immunohistochemical, HPLC and PET scan findings. Overall, the present study demonstrated that over-expression of HGF prevented neuronal death in a PD primate model, providing a potential novel therapy for PD.
\end{abstract}

Key Words: Gene therapy, naked DNA, HGF, parkinson disease, neurotrophic factor.

\section{INTRODUCTION}

Parkinson disease (PD) is a progressive neurodegenerative disorder that affects approximately 1,000,000 Americans [1]. The histopathological hallmarks of the disease are dopaminergic striatal insufficiency secondary to loss of dopaminergic neurons $[1,2]$. Clinically, PD is characterized by progressive abnormality including bradykinesia, limb rigidity and tremor $[1,2]$. As currently available treatment for PD has significant limitations, new therapeutic approaches are needed. Especially, treatment with neurotrophic factors ligands has become the center of interest, since a recent study demonstrated the clinical usefulness of recombinant glial cell-derived neurotrophic factor (GDNF) in PD patients [3]. Thus, it is important to further elucidate a ligand system(s) to limit the pathological changes of PD.

Hepatocyte growth factor (HGF) was identified as a potent mitogen for mature hepatocytes [4]. It is now well documented that HGF and its receptor, c-Met, signaling is critical for tissue protection and regeneration during embryogenesis and in parenchymal injury $[5,6]$. In brain tissue, HGF is expressed in non-parenchymal areas, while the c-Met/HGF receptor is found mainly on neurons [7]. In vitro, $\mathrm{HGF}$ is protective toward neural cells, including

*Address correspondence to this author at the Division of Clinical Gene Therapy, Graduate School of Medicine, Osaka University, 2-2 Yamada-oka, Suita 565-0871, Japan; Tel: +81-6-6879-3406; Fax: +81-6-6879-3409;

E-mail: ishida@cgt.med.osaka-u.ac.jp dopaminergic neurons $[7,8]$. In neural development, the HGF/c-Met system is critical for generation and maturation of neural cells [9]. In vivo, it has been revealed that HGF prevents delayed neural cell death in the ischemic brain and peripheral nervous system [10-14]. Furthermore, HGF prevents motor neuron cell death and improves the pathological changes in ALS [15].

Recent studies have further strengthened neurotrophic functions of HGF in pathological conditions: Sensory and sympathetic neurons and their precursors respond to HGF with increased differentiation, survival and axonal outgrowth [9]. HGF also cooperates with ciliary neurotrophic factor (CNTF) in promoting the survival and growth of parasympathetic and proprioceptive neurons [16], hence suggesting a ligand network(s) for protection of nervous systems. The broad spectrum of HGF activities and its synergy with other neurotrophic factors suggest that the major role of HGF is to potentiate the response of developing neurons to specific signals. Another benefit is that HGF is a potent angiogenic growth factor in various models including a brain hypoperfusion model $[17,18]$. More recently, we reported the results of a clinical trial in which intramuscular injection of naked HGF plasmid in patients with peripheral arterial disease was safe, feasible and could achieve successful improvement of ischemic limbs [19]. Based on these unique properties of HGF, we examined whether local injection of HGF cDNA could prevent the onset of $\mathrm{PD}$, using a non-human primate model. 


\section{MATERIALS \& METHODS}

\section{Plasmid DNA}

To produce an HGF expression vector, human HGF cDNA (2.2kb) [4] was inserted into a simple eukaryotic expression plasmid, pVAX1, that uses the cytomegalovirus (CMV) promoter/enhancer (pVAX1/HGF). The vector used as a control was the pcDNA3 expression vector plasmid, which was inserted into the reporter enzyme $\beta$-galactosidase (pcDNA3/lacZ). Plasmid DNA was purified with a QIAGEN plasmid isolation kit (Hilden, Germany).

\section{Transfection of HGF Plasmid}

Monkeys were anesthetized with sodium pentobarbital and placed in a stereotaxic instrument (Narishige, Tokyo, Japan). The skull was exposed and three holes were drilled over the right striatum. Then, $90 \mu \mathrm{l}$ naked human HGF plasmid DNA $(5 \mu \mathrm{g} / \mu \mathrm{l})$ or pcDNA3/LacZ plasmid DNA $(5 \mu \mathrm{g} / \mu \mathrm{l})$ was transfected unilaterally into nine sites in the right putamen using a Hamilton syringe. The sites of transfection were as follows: for track $1 ; 18.1 \mathrm{~mm}$ anterior, 11 $\mathrm{mm}$ lateral to the midline, and 15,17 and $19 \mathrm{~mm}$ below the surface of the brain: for track $2 ; 16.4 \mathrm{~mm}$ anterior, 11.5 $\mathrm{mm}$ lateral, 16, 18 and $20 \mathrm{~mm}$ depth: for track $3 ; 13.4 \mathrm{~mm}$ anterior, $12 \mathrm{~mm}$ lateral; 15,17 and $19 \mathrm{~mm}$ depth. At 7 days after transfection, a hemi-PD primate model (Macaca fascicularis, 2-3 kg) was produced by intra-arterial infusion of MPTP (right carotid artery) using X-ray angiography. A stock solution of MPTP-HCL $(1 \mathrm{mg}$ free base $/ \mathrm{ml})$ dissolved in $0.9 \% \mathrm{NaCl}$ was diluted to an appropriate volume so that the dose of MPTP to be administered was in a total volume of $40 \mathrm{ml} 0.9 \% \mathrm{NaCl}$; the solution was sterilized by filtration through a $22-\mu \mathrm{m}$ pore filter shortly before use. After animals were anesthetized using ketamine and xylazine at an intramuscular dose of $7 \mathrm{mg} / \mathrm{kg}$ and $0.6 \mathrm{mg} / \mathrm{kg}$, respectively, a catheter $(0.6 \mathrm{~mm}$, i.d.) was inserted using X-ray angiography via the right femoral artery, into the right internal carotid artery. MPTP was administered at $0.4 \mathrm{mg} / \mathrm{kg}$.

\section{Histological Evaluation, HPLC Analysis}

One week and 24 weeks after transfection of plasmid, primates were perfused transcardially with $0.9 \% \mathrm{NaCl}$ solution followed by $1000 \mathrm{ml}$ of $10 \%$ performaldehyde. The brain was dissected out, and postfixed overnight in the same solution. Tissues were analyzed by immunohistochemical staining. The brain was sectioned at a thickness of $6 \mu \mathrm{m}$ on a microtome, and blocked in PBS containing $1 \%$ bovine serum albumin. Primary antibodies were used as follows; antihuman HGF- $\beta$-chain (1:200; Institute of Immunology, Tokyo, Japan) and anti-tyrosine hydroxylase (TH) (1:400; Chemicon, Temecula, CA). These stains were developed by the avidin-biotin method (DAKO LSAB Kit, DAKO Japan, Kyoto, Japan) with diethylaminobenzidine as the chromogen. The number of TH-positive neurons in the SNi and ventral tegmental area (VTA) was counted under a light microscope (magnification, x200) in a blinded manner.

For biochemical assay in the caudate, putamen and substantia nigra, levels of dopamine and homovanillic acid (HVA) were measured using high performance liquid chromatography (HPLC) as described previouly [20].

\section{Behavioral Testing}

Animal behavior was assessed by videotaping home cage behavior under standardized conditions [21] from 4 to 24 weeks, every two weeks. At the beginning of each test, a technician entered the room and placed a small piece of fruit (apple or banana). The animal was then taped for $30 \mathrm{~min}$ with no one in the room. The technician then reentered the room to inject amphetamine $(2.0 \mathrm{mg} / \mathrm{kg})$ or apomorphine $(0.15 \mathrm{mg} / \mathrm{kg})$ subcutaneously to assess the functional change - amphetamine- and apomorphine-induced rotational asymmetry. Counterclockwise or clockwise rotation was observed for 1 hour. The tapes were coded and evaluated by trained raters. The rating scale used for hemiparkinsonian monkeys was as described previously [21].

\section{PET Analysis}

All animals underwent three PET scans with ${ }^{11} \mathrm{C}$-CFT, ${ }^{11} \mathrm{C}$-raclopride and ${ }^{18} \mathrm{~F}$-DOPA, and an MRI scan under anesthesia with continuous infusion of propofol $(5 \mathrm{mg} / \mathrm{kg} / \mathrm{hr})$. 2D or 3D dynamic PET acquisition (a total of $60 \mathrm{~min}$ for ${ }^{11} \mathrm{C}$-CFT and ${ }^{11} \mathrm{C}$-raclopride and $90 \mathrm{~min}$ for ${ }^{18} \mathrm{~F}$-dopa) was performed after intravenous administration of $5 \mathrm{mCi}$ tracer. Images were reconstructed as described previously [22]. For image analysis, 3D T1-weighted MRI images were also obtained with FSPGR sequence.

In ${ }^{11} \mathrm{C}$-CFT and ${ }^{11} \mathrm{C}$-raclopride PET, images of binding potential (BP) for each tracer were calculated based on a reference tissue model [23] and by pixel-by-pixel method [24] as well as the ratio of tracer delivery (RI) and efflux rate (k2), using the cerebellum as the reference region. In ${ }^{18} \mathrm{~F}$-dopa PET, images of dopa uptake constant, $\mathrm{Ki}$, were calculated by pixel-by-pixel based on a graphical method [25], using the cerebellum as the reference region. PET images were coregistered to MRI, and normalized to an MRI template in a standard space for monkey. We also obtained values in volumes of interest (VOI) in each side of the striatum as defined in the template MRI image.

\section{Statistical Analysis}

All values are expressed as mean \pm SEM. Analysis of variance with subsequent Duncan's test was used to determine the significance of differences in multiple comparisons. Differences with a $\mathrm{P}$ value less than 0.05 were considered significant.

\section{RESULTS}

In this study, we employed direct injection of plasmid DNA, although previous studies commonly used adenovirus and adeno-associated virus (AAV). However, there are serious safety problems such as immunogenicity, delayed demyelination, and difficulties in the preparation of a high titer of virus [26-28]. Since naked plasmid DNA is the most safe and easy to handle, intramuscular injection of naked plasmid DNA of angiogenic growth factors has been widely used clinically for the treatment of cardiovascular disease $[19,29]$. Although some researchers have tried to apply direct injection of naked plasmid DNA into the brain, the transfection efficiency was quite low [30]. To overcome this problem, the injection site was localized in a stereotaxic 
manner with delivery of a high dose. Aiming toward human gene therapy, we employed a hemi-parkinson primate to test the feasibility of gene therapy. Consistent with a rat study [31], transfection of human HGF plasmid DNA by direct injection into the primate putamen using a stereotaxic technique resulted in the detection of immunoreactive human HGF protein, while the untransfected contralateral side was not stained (Fig. 1a). Interestingly, exogenous HGF was mainly expressed in reactive astrocytes, and c-Met was also expressed in the injection sites [32]. Importantly, over $90 \%$ of dopaminergic neurons were lost in the PD model as assessed by staining with tyrosine hydroxylase, while most dopaminergic neurons survived in primates transfected with the human HGF gene (Fig. 1b). Survival of dopaminergic neurons was confirmed by the observation using HPLC that dopamine and HVA significantly remained in the brain in primates transfected with the human HGF gene as compared to control (Fig. 2, P<0.01).
Typical symptoms of PD such as tremor and rigidity of the limbs of primates gradually increased after the onset of PD. In contrast, transfection of human HGF gene attenuated the clinical symptoms of $\mathrm{PD}(\mathrm{P}<0.01$, Fig. 3a, b, c). Similar to the rat model, the hemi-parkinson primate model without treatment and transfected with control vector demonstrated amphetamine- or apomorphine-induced rotation from 4 to 19 weeks after MPTP treatment, while normal primates did not rotate either clockwise or counterclockwise. The number of amphetamine- or apomorphine-induced rotations in the PD model was significantly inhibited in primates transfected with the human HGF gene as compared to control (Fig. 4).

Finally, we confirmed the remaining function of dopaminergic neurons using PET. Using PET with ${ }^{18} \mathrm{~F}$-labeled F-DOPA, which evaluates the function of dopaminergic neurons, uptake of ${ }^{18} \mathrm{~F}$-FDOPA was lost in monkeys transfected with control vector, while uptake of ${ }^{18}$ F-FDOPA was

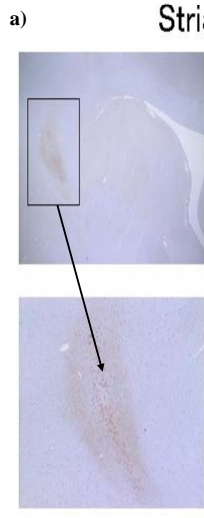

HGF (Right)
Striatum

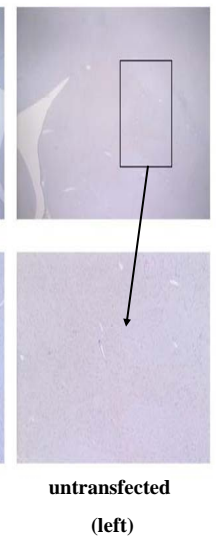

b)

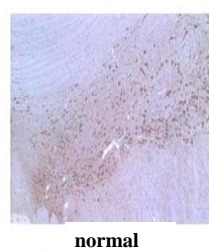

normal

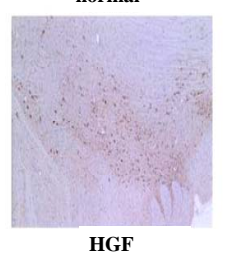

HGF
SNc

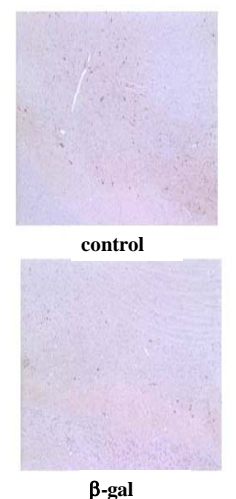

Fig. (1). (a) Immunohistochemical staining of human immunoreactive HGF protein at 1 week after transfection in primate striatum HGF plasmid DNA was transfected into the right side of the PD primate model, while the left side was not transfected. HGF (right) $=$ right side of PD model primate transfected with HGF gene, untransfected (left) = left side (untransfected area) of PD model primate transfected with HGF gene. b) Immunohistochemical staining of tyrosine hydroxylase at 24 weeks after transfection in primate striatum Normal $=$ untransfected sham-operated primates, Control $=$ untransfected PD model primates, HGF $=$ PD primates transfected with human HGF gene, $\beta$-gal $=$ PD primates transfected with $\beta$-galcatosidase gene.

Dopamine

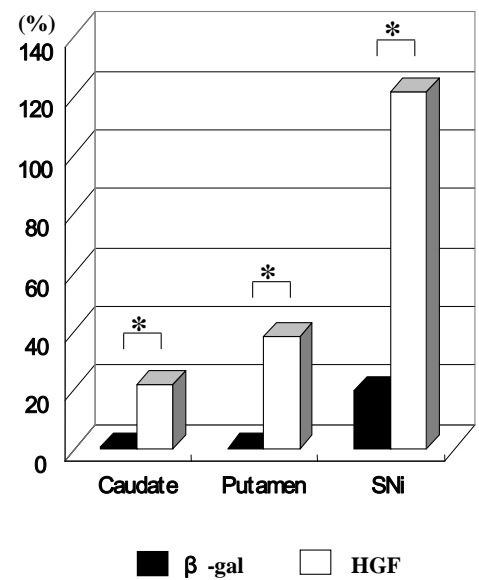

HVA

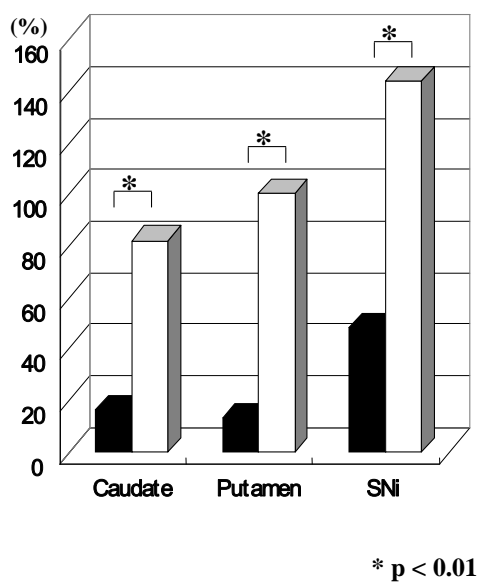

Fig. (2). Analysis of dopamine and HVA content by HPLC at 24 weeks after transfection HVA=homovanillic acid, final metabolic product of dopamine. $\beta$-gal $=$ PD primates transfected with $\beta$-galcatosidase gene, $\mathrm{HGF}=\mathrm{PD}$ primates transfected with human $\mathrm{HGF}$ gene. $\mathrm{n}=3$ for each group. 

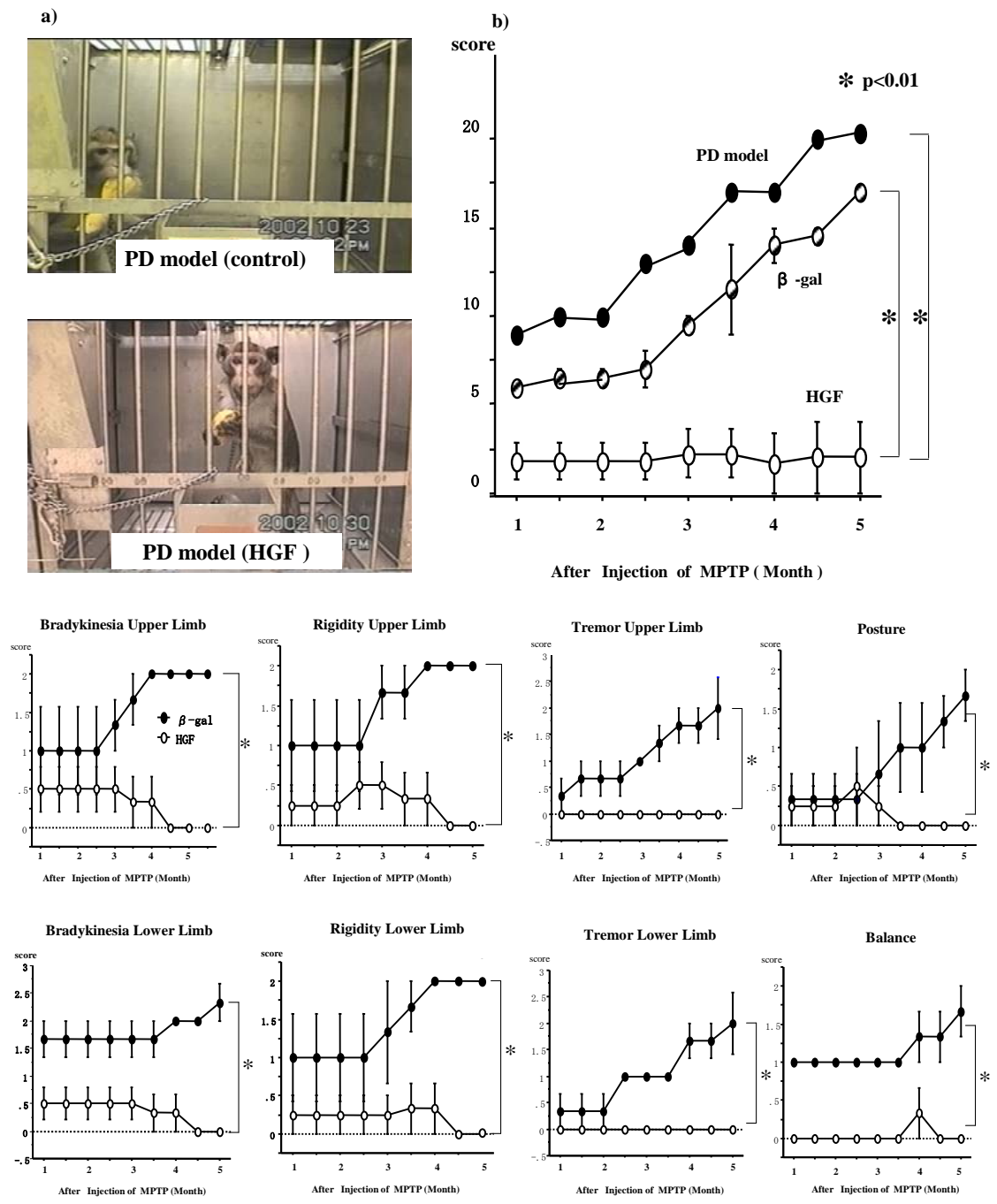

c)

Fig. (3). (a) Typical example of appearance of primate at 12 weeks after transfection PD model (control) $=$ PD primates transfected with $\beta$ galcatosidase gene, PD model (HGF) = PD primates transfected with human HGF gene. b) Reduction of abnormal symptom score by HGF gene transfer after MPTP treatment PD model $=$ untransfected PD model primates, $\beta$-gal $=$ PD primates transfected with $\beta$-galactosidase gene, HGF $=$ PD primates transfected with human HGF gene. $* \mathrm{P}<0.01$ vs. HGF. $\mathrm{n}=5$ for each group. c) Effect of HGF gene transfer on various clinical symptoms in PD primates $\beta$-gal $=\mathrm{PD}$ primates transfected with $\beta$-galactosidase gene, $\mathrm{HGF}=\mathrm{PD}$ primates transfected with human HGF gene. ${ }^{*} \mathrm{P}<0.01$ vs. HGF. $\mathrm{n}=5$ for each group.

detected in primates transfected with the human HGF gene (Fig. 5). Similar results were obtained using ${ }^{11} \mathrm{C}-\mathrm{CFT}$ as a marker of the dopamine transporter. In contrast, the dopamine D2 receptor as assessed by ${ }^{11} \mathrm{C}$-raclopride, was upregulated in $\mathrm{PD}$ primates transfected with control vector (Fig. 5), while no obvious difference could be detected in PD primates transfected with human HGF gene. As dysphasia, paresis, or nausea due to severe damage in the striatum, we measured body weight after gene transfer. Primates transfected with naked plasmid DNA showed no difference in body weight, histological staining in the striatum and behavioral abnormality (data not shown).

\section{DISCUSSION}

$\mathrm{PD}$ is a progressive neurodegenerative disorder that affects approximately 1,000,000 Americans [1]. Its cause remains unknown. The histopathological hallmarks of the disease are dopaminergic striatal insufficiency secondary to a loss of dopaminergic neurons in the substantia nigra pars compacta and intracellular inclusions called Lewy bodies [1, 2]. As all currently available symptomatic treatment for PD has significant limitations, new therapeutic approaches are needed. Gene therapy might be a candidate for delivering therapeutic molecules to site-specific regions of the central nervous system. Thus, researchers have tried to establish effective gene therapy, including the use of tyrosine hydroxylase and GDNF. Especially, treatment with neurotrophic factors has become the center of interest, as a recent study demonstrated the clinical usefulness of recom-binant GNDF in PD patients [3]. Gene therapy has the following advantages as compared to recombinant therapy: 1) It has the potential to maintain an optimally high, local concentration over time. It may be preferable to deliver a lower dose over a period of several days or more from an actively expressed transgene rather than a single or multiple doses of recombinant protein, to avoid side effects, and 2) regarding 
a) amphetamine

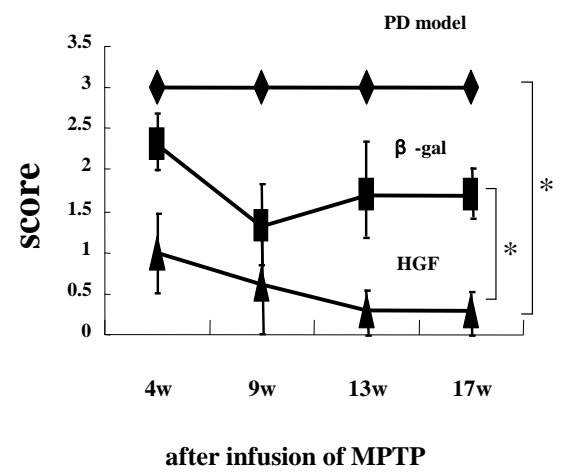

b)

apomorphine

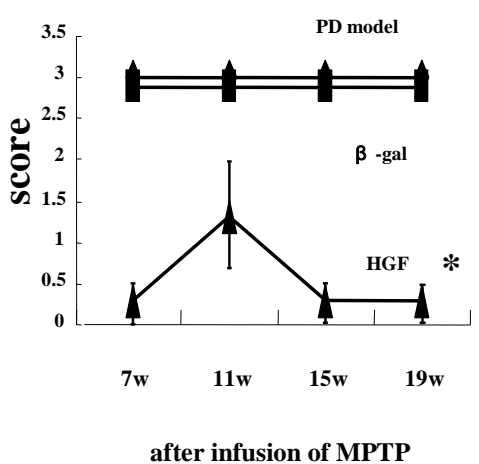

Fig. (4). Inhibition of a) amphetamine- and b) apomorphine-induced rotational behavior from 4 to 19 weeks after MPTP treatment PD model $=$ untransfected PD model primates, $\beta$-gal $=\mathrm{PD}$ primates transfected with $\beta$-galactosidase gene, HGF $=$ PD primates transfected with human HGF gene. $* \mathrm{P}<0.01$ vs. HGF. $\mathrm{n}=5$ for each group. Score: $0=$ no rotation, $1=$ only twists head and body, $2=$ temporary rotation, $3=$ continuous rotation.

${ }^{18}$ F-FDOPA
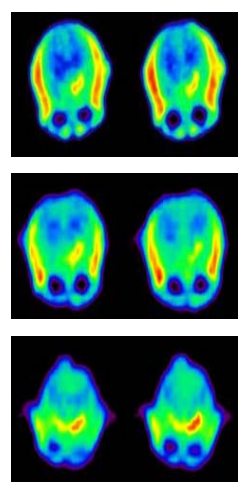

${ }^{11}$ C-CFT
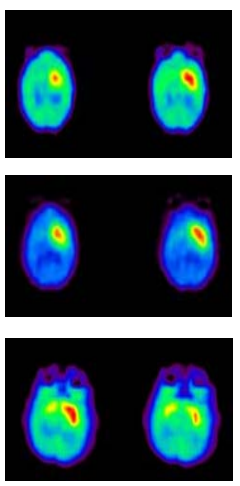

${ }^{11}$ C-raclopride

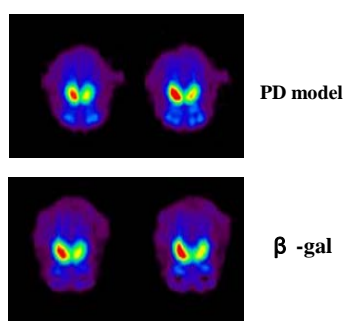

$\infty$

Fig. (5). PET analysis of ${ }^{18}$ FDOPA (dopamine synthesis), ${ }^{11} \mathrm{C}$-CFT (dopamine transporter) and ${ }^{11} \mathrm{C}$-raclopride (dopamine D2 receptor) at 24 weeks after transfection PD model $=$ untransfected $\mathrm{PD}$ model primates, $\beta$-gal $=\mathrm{PD}$ primates transfected with $\beta$-galactosidase gene, $\mathrm{HGF}=\mathrm{PD}$ primates transfected with human HGF gene. $\mathrm{n}=3$ for each group.

economics, it needs to be considered which therapy would ultimately cost less to develop, implement, and reimburse, particularly for those indications requiring multiple or even protracted treatment.

In addition to GDNF, the present study demonstrated HGF to be a potential candidate for the treatment of PD. Both HGF and the c-Met/HGF receptor of membranespanning tyrosine kinase are expressed in various regions of the brain [7]. It is reported that sympathetic neurons of the superior cervical ganglion co-express bioactive HGF and c-Met both in vivo and in vitro [9]. Exogenous HGF selectively promotes the growth of cultured sympathetic neurons; the magnitude of this growth effect is similar to that observed with exogenous nerve growth factor (NGF) [9]. In addition, recent studies suggest that HGF prevents apoptosis in cerebellar granular neurons via the phosphatidylinositol-3 (PI3)-kinase/Akt pathway [33]. It is also reported that HGF cooperates with ciliary neurotrophic factor (CNTF) in promoting the survival and growth of parasympathetic and proprioceptive neurons, and that within the same neurons, the effects of HGF on survival and growth are selectively dependent on which other signaling pathways are concurrently activated [16]. Given these unique properties, HGF is considered to be a novel neuroprotective agent. Indeed, administration of recombinant HGF as well as HGF gene therapy has been reported to protect hippocampal neurons from ischemia-induced delayed neuronal death [10, 12]. In addition, a recent study provided a strong rationale for the potential clinical use of HGF for the treatment of motor neuron degenerative disease, as intramuscular injection of naked HGF plasmid in patients with peripheral arterial disease is safe, feasible and can achieve successful improvement of ischemic limbs [13]. Based upon these studies, we examined the possibility that gene transfer of HGF into the striatum could have beneficial effects on dopaminergic neuronal death in a non-human primate PD model.

Our present study clearly demonstrated that 1) abnormal rotation of hemi-PD primates could be prevented by local transfection of human HGF into the striatum in a stereotaxic manner, 2) clinical symptoms such as tremor were also improved by HGF gene therapy, while the clinical symptoms gradationally worsened in control PD primates, and 3) func- 
tional analysis by PET clearly demonstrated remaining dopaminergic neurons, consistent with HPLC analysis. This study demonstrated the therapeutic potential of HGF in a hemi-PD primate model, and we also obtained similar results in a rat PD model [31]. While further studies after the onset of PD are necessary for progressing toward human therapy, HGF might have therapeutic value to treat PD. One important point of the present study is the use of plasmid DNA in gene therapy, although numerous previous studies commonly used adenovirus or adeno-associated virus (AAV). However, there are serious safety problems such as immunogenicity, delayed demyelination, and difficulties in the preparation of a high titer of virus in the case of viral vectors [26-28]. Since naked plasmid DNA is very safe and easy to handle as compared to viral vectors, intramuscular injection of naked plasmid DNA of angiogenic growth factors such as vascular endothelial growth factor (VEGF) has been widely used clinically for the treatment of ischemic cardiovascular disease. Although the transfection efficiency using direct injection of naked plasmid DNA into the brain was low, delivery of a high dose into a localized injection site in a stereotaxic manner might overcome the low efficiency, consistent with a previous report [30]. Indeed, the exogenous protein of human HGF was readily detected in the striatum. Interestingly, our previous study demonstrated that exogenous HGF was mainly expressed in reactive astrocytes, and that c-Met was also expressed in the injection site [32].

Overall, the present study demonstrated that gene transfer of HGF protected dopaminergic neurons from toxicity induced by MPTP, resulting in prevention of the onset of PD in a non-human primate model. As no significant loss of body weight or histological change was observed throughout the experimental period, these data demonstrate the clinical utility of the therapeutic strategy based upon plasmid DNAmediated transfer. What is the clinical relevance of HGF gene transfer based upon plasmid DNA? First, it is possible to decrease the potential side effects and cost. Second, avoiding a viral gene transfer method such as use of an adenovirus may increase the safety of gene therapy and extend its application to a wide variety of target diseases. Third, further modification of delivery tools such as the catheter may expand the utility of the present method of transfection in the progress toward human gene therapy. Fourth, as expected, an in vivo study demonstrated no apparent toxicity in non-human primates. In addition to its neurotrophic action, HGF is a potent angiogenic growth factor, so stimulation of local blood flow around the striatum might become a new therapeutic option to treat Parkinson syndrome induced by common adult diseases such as hypertension and diabetes.

\section{ACKNOWLEDGEMENTS}

This work was partially supported by a Grant-in-Aid from the Organization for Pharmaceutical Safety and Research, a Grant-in-Aid from The Ministry of Public Health and Welfare, and the Ministry of Education, Culture, Sports, Science and Technology, the Japanese Government.

\section{COMPETING INTERESTS STATEMENT}

Ryuichi Morishita has stocks for AnGes MG and serves as Board Member of AnGes MG which developed HGF.

\section{REFERENCES}

[1] Schrag A, Schott JM. Epidemiological, clinical, and genetic characteristics of early-onset parkinsonism. Lancet Neurol 2006; 5: 35563. Review.

[2] Clarke C, Moore AP. Parkinson's disease. Clin Evid 2005; 13: 1658-77. Review.

[3] Ill SS, Patel NK, Hotton GR, et al. Direct brain infusion of glial cell line-derived neurotrophic factor in Parkinson disease. Nat Med 2003; 9: 589-95.

[4] Nakamura T, Nishizawa T, Hagiya M, et al. Molecular cloning and expression of human hepatocyte growth factor. Nature 1989; 342 : 440-43.

[5] Funakoshi H, Nakamura T. Hepatocyte growth factor: from diagnosis to clinical applications. Clin Chim Acta 2003; 327: 1-23.

[6] Birchmeier C, Gherardi E. Developmental roles of HGF/SF and its receptor, the c-Met tyrosine kinase. Trends Cell Biol 1998; 8: 404-10.

[7] Honda S, Kagoshima M, Wanaka A, Tohyama M, Matsumoto K, Nakamura T. Localization and functional coupling of HGF and c-Met/HGF receptor in rat brain: implication as neurotrophic factor. Brain Res Mol Brain Res 1995; 32(2): 197-210.

[8] Hamanoue M, Takemoto N, Matsumoto K, Nakamura T, Nakajima K, Kohsaka S. Neurotrophic effect of hepatocyte growth factor on central nervous system neurons in vitro. J Neurosci Res 1996; 43(5): 554-64.

[9] Maina F, Klein R. Hepatocyte growth factor, a versatile signal for developing neurons. Nat Neurosci 1999; 2: 213-7.

[10] Miyazawa T, Matsumoto K, Ohmichi H, Katoh H, Yamashima T, Nakamura T. Protection of hippocampal neurons from ischemiainduced delayed neuronal death by hepatocyte growth factor: a novel neurotrophic factor. J Cereb Blood Flow Metab 1998; 18(4): 345-8.

[11] Date I, Takagi N, Takagi K, et al. Hepatocyte growth factor improved learning and memory dysfunction of microsphereembolized rats. J Neurosci Res 2004; 78(3): 442-53.

[12] Hayashi K, Morishita R, Nakagami H, et al. Gene therapy for preventing neuronal death using hepatocyte growth factor: in vivo gene transfer of HGF to subarachnoid space prevents delayed neuronal death in gerbil hippocampal CA1 neurons. Gene Ther 2001; 8: 1167-73.

[13] Koike H, Morishita R, Iguchi S, et al. Enhanced angiogenesis and improvement of neuropathy by cotransfection of human hepatocyte growth factor and prostacyclin synthase gene. FASEB J 2003; 17 : 779-81.

[14] Shimamura M, Sato N, Oshima K, et al. Novel therapeutic strategy to treat brain ischemia: overexpression of hepatocyte growth factor gene reduced ischemic injury without cerebral edema in rat model. Circulation 2004; 109(3): 424-31.

[15] Sun W, Funakoshi H, Nakamura T. Overexpression of HGF retards disease progression and prolongs life span in a transgenic mouse model of ALS. J Neurosci 2002; 22(15): 6537-48.

[16] Davey F, Hilton M, Davies AM. Cooperation between HGF and CNTF in promoting the survival and growth of sensory and parasympathetic neurons. Mol Cell Neurosci 2000; 15: 79-87.

[17] Yoshimura S, Morishita R, Hayashi K, et al. Gene transfer of hepatocyte growth factor to subarachnoid space in cerebral hypoperfusion model. Hypertension 2002; 39(5): 1028-34.

[18] Tomita N, Morishita R, Taniyama Y, et al. Angiogenic property of hepatocyte growth factor is dependent on upregulation of essential transcription factor for angiogenesis, ets-1. Circulation 2003; 107(10): 1411-17.

[19] Morishita R, Aoki M, Hashiya N, et al. Safety evaluation of clinical gene therapy using hepatocyte growth factor to treat peripheral arterial disease. Hypertension 2004; 44(2): 203-9.

[20] Bankiewicz KS, Oldfield EH, Chiueh CC, Doppman JL, Jacobowitz DM, Kopin IJ. Hemiparkinsonism in monkeys after unilateral internal carotid artery infusion of 1-methyl-4-phenyl-1,2,3,6tetrahydropyridine (MPTP). Life Sci 1986; 39(1): 7-16.

[21] Ovadia A, Zhang Z, Gash DM. Increased susceptibility to MPTP toxicity in middle-aged rhesus monkeys. Neurobiol Aging 1995; 16(6): $931-7$

[22] Hayashi T, Ohnishi T, Okabe S, et al. Long-term effect of motor cortical repetitive transcranial magnetic stimulation [correction]. Ann Neurol 2004; 56(1): 77-85. Erratum in: Ann Neurol 2004; 56(2): 311 . 
[23] Lammertsma AA, Hume SP. Simplified reference tissue model for PET receptor studies. Neuroimage 1996; 4(3 Pt 1): 153-8.

[24] Gunn RN, Lammertsma AA, Hume SP, Cunningham VJ. Parametric imaging of ligand-receptor binding in PET using a simplified reference region model. Neuroimage 1997; 6(4): 279-87.

[25] Patlak CS, Blasberg RG, Fenstermacher JD. Graphical evaluation of blood-to-brain transfer constants from multiple-time uptake data. J Cereb Blood Flow Metab 1983; 3(1): 1-7.

[26] Schnell MA, Zhang Y, Tazelaar J, et al. Activation of innate immunity in nonhuman primates following intraportal administration of adenoviral vectors. Mol Ther 2001; 3: 708-22.

[27] Dewey RA, Morrissey G, Cowsill CM, et al. Chronic brain inflammation and persistent herpes simplex virus 1 thymidine kinase expression in survivors of syngeneic glioma treated by adenovirus-mediated gene therapy: implications for clinical trials. Nat Med 1999; 5: 1256-63.
[28] Hsich G, Sena-Esteves M, Breakfield XO. Critical issues in gene therapy for neurologic disease. Hum Gene Ther 2002; 13: 579-604.

[29] Baumgartner I, Rauh G, Pieczek A, et al. Lower-extremity edema associated with gene transfer of naked DNA encoding vascular endothelial growth factor. Ann Intern Med 2000; 132: 880-84.

[30] Schwartz B, Benoist C, Abdallah B, et al. Gene transfer by naked DNA into adult mouse brain. Gene Ther 1996; 3: 405-11.

[31] Koike H, Ishida A, Shimamura M, et al. Prevention of onset of parkinson's disease by in vivo gene transfer of human hepatocyte growth factor in rodent model: A model of gene therapy for parkinson's disease. Gene Ther 2006; 23:1639-44.

[32] Sekiguchi K, Yasuzumi F, Morishita R. Exogenous expression of hepatocyte growth factor (HGF) in rat striatum by naked plasmid DNA. Neurosci Res 2003; 45: 173-80.

[33] Zhang L, Himi T, Morita I, Murota S. Hepatocyte growth factor protects cultured rat cerebellar granule neurons itol-3 kinase/Akt pathway. J Neurosci Res 2000; 59(4): 489-96.

(C) Koike et al.; Licensee Bentham Open .

This is an open access article licensed under the terms of the Creative Commons Attribution Non-Commercial License (http://creativecommons.org/licenses/by-nc/3.0/) which permits unrestricted, non-commercial use, distribution and reproduction in any medium, provided the work is properly cited. 